\title{
AZIONI INFERMIERISTICHE NELLE CURE PRIMARIE IN CONSIDERAZIONE DEI RISCHI DI IPERTENSIONE DURANTE LA GRAVIDANZA
}

\section{REVISIONE ARTICOLO}

ADRIÃO, Iracely Santos ${ }^{1}$, BARBOSA, Marluce Sampaio Nobre $^{2}$

ADRIÃO, Iracely Santos. BARBOSA, Marluce Sampaio Nobre. Azioni infermieristiche nelle cure primarie in considerazione dei rischi di ipertensione durante la gravidanza. Revista Científica Multidisciplinar Núcleo do Conhecimento. Anno 06, Ed. 09, Vol. 04, pp. 84-100. Settembre 2021. ISSN: 2448-0959, Link di accesso: https://www.nucleodoconhecimento.com.br/salute/rischi-di-ipertensione, DOI: 10.32749/nucleodoconhecimento.com.br/salute/rischi-di-ipertensione

\section{RIEPILOGO}

La preeclampsia si presenta come una delle sindromi ipertensive che possono colpire le donne durante la gravidanza, durante il parto e/o nel periodo postpartum fino a 10 giorni, tanto che è ancora una patologia che, se non adeguatamente diagnosticata e trattata, può causare gravi danni e persino portare alla morte della donna incinta e/o del parto. L'obiettivo principale di questo studio è conoscere la prevenzione della preeclampsia nelle cure primarie da parte dell'allattamento. Pertanto, la seguente domanda è: quali sono le azioni infermieristiche nelle cure primarie di fronte ai rischi di ipertensione durante la gravidanza? II presente lavoro si basa su una ricerca bibliografica, con un approccio qualitativo condotto attraverso il metodo della revisione integrativa. Per raccogliere i dati, è stata condotta un'indagine nei database: Google Scholar, Pubmed, Scielo e Medline. I descrittori utilizzati erano: Rischi. Gestazione. Ipertensione. Allattamento. I criteri di inclusione utilizzati per la selezione dei campioni sono stati: articoli disponibili per via elettronica pubblicati dal 2015 al 2020; lingua portoghese. I criteri di esclusione erano articoli inferiori al 2015 e parole chiave che

\footnotetext{
${ }^{1}$ Studentessa in Infermieristica presso I'Università CEUMA.

${ }^{2}$ Master in Malattie Tropicali.

RC: 99509

Link di accesso: https://www.nucleodoconhecimento.com.br/salute/rischi-di-ipertensione
} 
non sono correlate al tema. Per l'analisi completa degli articoli selezionati, è stata utilizzata una piattaforma online (ricerca nei database), con lo scopo di estrarre (titolo e scopo degli articoli), organizzare (in forma di tabella), sumarizzare le informazioni e facilitare la formazione del database. I risultati hanno indicato 35 articoli pubblicati, ma solo 15 articoli, negli ultimi cinque anni, hanno come tema principale la prevenzione e i rischi della preeclampsia nelle cure primarie. Si conclude che le principali condotte utilizzate non solo dagli infermieri, ma dall'intero team che lavora nelle cure primarie, dovrebbero essere basate sul processo di accoglienza incentrato su azioni che possono essere determinanti nella prevenzione della preeclampsia.

Parole chiave: Rischi, Gravidanza, Ipertensione, Allattamento.

\section{INTRODUZIONE}

Attualmente viviamo in un mondo profondamente segnato dalla produzione di conoscenza in tutti i settori, compresa l'assistenza infermieristica e la medicina. Anche con questo, il numero di donne affette da malattie ampiamente conosciute, come la sindrome da gravidanza ipertensiva specifica (SHEG), è ancora molto grande. La sua incidenza è elevata, al punto da essere trattata come un problema di salute pubblica (ARAÚJO, et al, 2017).

Se le sindromi ipertensiva legate allo stato di gravidanza delle donne sono così importanti, quindi, devono essere conosciute dalle autorità e dagli operatori sanitari, e quindi è da chiedersi, perché nel $21^{\circ}$ secolo, tali patologie compromettono ancora la salute e spesso anche la vita delle donne incinte e dei neonati. Quando è noto che la pratica degli esami prenatali identificherebbe il problema, o almeno consentirebbe la sua diagnosi precoce, che in pratica consentirebbe un intervento tempestivo (ARNALDO; CARDOZO, 2021).

Tutte queste riflessioni hanno contribuito e motivato la scelta del tema come oggetto di studio, poiché la sua fattibilità è stata in parte il seguente problema: c'è stato un alto tasso di donne in gravidanza che sono quasi sempre curate a presentare segni e sintomi di SHEG, in particolare preeclampsia. Quindi, quali sono le azioni 
infermieristiche in Primary Care in considerazione dei rischi di ipertensione durante la gravidanza? E come risposta provvisoria al problema affermato, è stata adottata l'ipotesi che le donne nel periodo gestazionale e persino parturiente e nel periodo postpartum, presentino un alto indice di preeclampsia, perché non hanno avuto un adeguato follow-up prenatale (ARAÚJO et al., 2017).

Gli studi che coinvolgono questo insieme di informazioni (rischi di ipertensione durante la gravidanza), aiutano nella prevenzione dell'ipertensione, poiché stimola e avvisa la popolazione ad adottare cambiamenti nelle abitudini alimentari e nella pratica abituale dell'attività fisica, oltre a facilitare l'approccio farmacologico dei suoi componenti isolati o della sindrome metabolica stessa, attraverso professionisti e ricercatori (ARNALDO; CARDOZO, 2021).

La scelta del tema agisce per portare alle donne incinte informazioni che le portano a una vita più sana, perché questo è il compito principale degli operatori sanitari. $E$ per queste donne in gravidanza dovrebbe iniziare a prendere al loro giorno per giorno una dieta più sana e naturale, avendo come consiglio il consumo di frutta, verdura, verdura. Essere orientati a ridurre al minimo il consumo di carne, grassi, cibi fritti, panini e altre forme di alimenti dannosi per la salute e che portano ad un aumento dell'ipertensione (SOARES et al., 2015).

Per una buona qualità della vita, la donna incinta considerata ipertesa ha bisogno di mantenere una vita sana, perché molti di questi aderiscono al trattamento farmacologico, in cui la pressione sanguigna è controllata attraverso l'ingestione di compresse. Esiste anche un trattamento non farmacologico che viene eseguito attraverso pratiche di attività fisica, che può anche essere un complemento al trattamento farmacologico (MELO et al., 2015).

La pratica dell'esercizio fisico è di fondamentale importanza per la salute e la qualità della vita in generale e soprattutto per le donne in gravidanza, che hanno l'abitudine di condurre una vita sedentaria, poiché si trova nel periodo di riposo della professione, e inizia uno stile di vita sedentario (SOARES et al., 2015). 
Il lavoro è giustificato perché è di grande interesse per l'allattamento e la donna che intende essere madre. L'interesse per la ricerca è stato motivato anche dal fatto che questo è un tema che porta informazioni importanti e fondamentali ad ogni donna che mira a rimanere incinta e per l'area di attività infermieristica.

E così la ricerca porta insegnamenti a una buona qualità della vita delle donne in gravidanza, dal momento che le donne ipertese hanno bisogno di mantenere una vita sana, perché molte di queste aderiscono al trattamento farmacologico, dove la pressione sanguigna è controllata attraverso l'ingestione di compresse. Esiste anche un trattamento non farmacologico che viene effettuato attraverso pratiche di attività fisica, che può anche essere un complemento al trattamento farmacologico (SANTOS; NETO, 2016).

L'obiettivo principale di questo lavoro è conoscere le condotte di prevenzione della preeclampsia nelle cure primarie da parte dell'assistenza infermieristica.

\section{METODOLOGIA}

Per quanto riguarda le procedure metodologiche del presente studio, è stato utilizzato lo sviluppo della ricerca bibliografica, ovvero una revisione della letteratura di articoli scientifici sull'ipertensione e la gravidanza. Per la presente ricerca scientifica, gli articoli sono stati ricercati nei database di Google Scholar, Pubmed, Scielo e Medline, libri di riviste sanitarie e articoli scientifici, sulla base di materiali già elaborati.

Inoltre, la ricerca esplorativa per Lakatos e Marconi (2010, p. 171) "gli studi combinati esplorativo-descrittivo hanno l'obiettivo di descrivere un fenomeno completamente certo, come lo studio di un caso per il quale vengono eseguite analisi empiriche e teoriche".

Pertanto, la ricerca cerca sempre la migliore approssimazione del ricercatore con la situazione reale dei problemi, inoltre, consiste nel comprendere in modo meno totalitario, frammentando le opinioni presentate e mirando alla prospettiva della prospettiva degli attori coinvolti nel processo. 
In relazione ai mezzi, le procedure di ricerca bibliografica sono state applicate a questo studio. Pertanto, pubblicazioni nazionali, in libri, articoli autori di professionisti infermieristici e riviste faranno parte della costruzione di questo materiale (FONSECA, 2002).

Pertanto, è evidente che, nonostante tutti gli sforzi per sollevare le opinioni degli attori sociali coinvolti nel processo a cui la ricerca si propone di studiare, si percepisce che la base teorica attraverso l'indagine bibliografica diventa la base per la comprensione della ricerca.

In questo studio, la comprensione di alcuni autori è stata sollevata sulla base di ricerche bibliografiche in dettaglio alla ricerca del punto di vista di questo studio. Arricchente nelle conoscenze, perché è di fondamentale importanza verificare la posizione di questi autori riguardo all'allattamento e ai rischi di ipertensione durante la gravidanza.

Pertanto, i criteri di inclusione erano: articoli pubblicati in portoghese, che affrontano I'allattamento e i rischi di ipertensione durante la gravidanza e che sono stati pubblicati e indicizzati in questi database dal 2015 al 2020. I criteri di esclusione erano articoli inferiori al 2015 e parole chiave che non sono correlate al tema. Le parole chiave utilizzate sono state: Rischi. Gestazione. Ipertensione. Allattamento. La raccolta dei dati è stata effettuata anche nella prima metà del 2021.

La presente ricerca ha avuto tra le sue fonti di raccolta dati, attraverso la catalogazione e il raccoglitore di documentazione bibliografica, libri, articoli, riviste, giornali, periodici e articoli pubblicati su Internet, di autori contemporanei, attraverso i quali sono state eseguite diverse interpretazioni del tema. Pertanto, è importante menzionare, il mezzo di raccolta dei dati correlati, è ciò che ci si aspetta di coltivare nel miglior modo possibile le origini e le motivazioni delle risposte trovate o meno alla fine della ricerca.

\section{SVILUPPO}

Il campione finale di questa revisione consisteva in 15 (quindici) articoli scientifici, selezionati in base a criteri di inclusione precedentemente stabiliti. La tabella 1 RC: 99509

Link di accesso: https://www.nucleodoconhecimento.com.br/salute/rischi-di-ipertensione 
rappresenta le specifiche di ciascuno degli articoli, distribuite in base a: anno; periodico; nome e titolo degli autori.

Tavolo uno. Elenco degli studi selezionati per anno, rivista, autori e titolo tra il 2015 e il 2020.

\begin{tabular}{|c|c|c|c|}
\hline ANNO & PERIODICO & AUTORI & TITOLO \\
\hline 2017 & $\begin{array}{l}\text { Rev enferm UFPE } \\
\text { on line }\end{array}$ & $\begin{array}{l}\text { ARAÚJO, Isabella } \\
\text { Félix Meira, et al }\end{array}$ & $\begin{array}{l}\text { Síndromes hipertensivas e } \\
\text { fatores de risco associados à } \\
\text { gestação }\end{array}$ \\
\hline 2021 & $\begin{array}{l}\text { Revista científica } \\
\text { multidisciplinar } \\
\text { núcleo do } \\
\text { conhecimento }\end{array}$ & $\begin{array}{l}\text { ARNALDO, } \\
\text { de } \\
\text { CARDOriany } \\
\text { Maryanne } \\
\text { Freire }\end{array}$ & $\begin{array}{l}\text { Assistência de enfermagem } \\
\text { às gestantes hipertensas na } \\
\text { prevenção da prematuridade: } \\
\text { revisão bibliográfica }\end{array}$ \\
\hline 2019 & $\begin{array}{ll}\text { Trabalho } & \text { de } \\
\text { Conclusão } & \text { de } \\
\text { Curso } & \end{array}$ & $\begin{array}{l}\text { CABRAL, Gustavo } \\
\text { Pereira }\end{array}$ & $\begin{array}{l}\text { Cuidados de enfermagem a } \\
\text { mulheres com doença } \\
\text { hipertensiva específica da } \\
\text { gestação: uma revisão } \\
\text { integrativa }\end{array}$ \\
\hline 2017 & $\begin{array}{l}\text { International } \\
\text { Nursing Congress }\end{array}$ & $\begin{array}{l}\text { ENDRINGER, Deyvid } \\
\text { Dantas; } \quad \text { CRUZ, } \\
\text { Monielle Lima }\end{array}$ & $\begin{array}{l}\text { Representatividade do } \\
\text { enfermeiro na assistência a } \\
\text { gestantes com pré-eclâmpsia }\end{array}$ \\
\hline 2015 & $\begin{array}{l}\text { REBES - } \\
\text { Brasileira de } \\
\text { Educação e Saúde }\end{array}$ & $\begin{array}{l}\text { MELO, Wyara } \\
\text { Ferreira, et al }\end{array}$ & $\begin{array}{l}\text { A hipertensão gestacional e o } \\
\text { risco de pré-eclampsia: } \\
\text { revisão bibliográfica }\end{array}$ \\
\hline 2018 & $\begin{array}{l}\text { Brazilian Journal of } \\
\text { Surgery and } \\
\text { Clinical Research - } \\
\text { BJSCR }\end{array}$ & $\begin{array}{l}\text { OLIVEIRA, Leilyanne } \\
\text { de Araújo Mendes, et } \\
\text { al }\end{array}$ & $\begin{array}{l}\text { Cuidados de enfermagem a } \\
\text { gestante com síndrome } \\
\text { hipertensiva: } \\
\text { integrativa. }\end{array}$ \\
\hline 2018 & $\begin{array}{l}\text { Revista Científica } \\
\text { Multidisciplinar }\end{array}$ & $\begin{array}{l}\text { SANTOS, } \\
\text { Carvalho. }\end{array}$ & $\begin{array}{l}\text { Enfermagem e os cuidados } \\
\text { Emergenciais na doença }\end{array}$ \\
\hline
\end{tabular}




\begin{tabular}{|c|c|c|c|}
\hline & $\begin{array}{l}\text { Núcleo do } \\
\text { Conhecimento }\end{array}$ & $\begin{array}{l}\text { BRASILEIRO, } \\
\text { Marislei Espíndula }\end{array}$ & $\begin{array}{l}\text { hipertensiva específica na } \\
\text { gravidez }\end{array}$ \\
\hline 2016 & $\begin{array}{l}\text { Revista Científica } \\
\text { da FASETE }\end{array}$ & $\begin{array}{l}\text { SANTOS, Joelma } \\
\text { Oliveira Silva; NETO, } \\
\text { Thiago Paulo de } \\
\text { Almeida }\end{array}$ & $\begin{array}{l}\text { Atuação do enfermeiro na } \\
\text { redução da ocorrência da } \\
\text { pré-eclâmpsia: uma revisão } \\
\text { integrativa }\end{array}$ \\
\hline 2020 & Enfermagem Brasil & $\begin{array}{l}\text { SARMENTO, Rayani } \\
\text { Silva, et al }\end{array}$ & $\begin{array}{l}\text { Pré-eclâmpsia na gestação: } \\
\text { ênfase na assistência de } \\
\text { enfermagem }\end{array}$ \\
\hline 2016 & $\begin{array}{l}\text { Centro } \\
\text { Universitário Ritter } \\
\text { dos Reis }\end{array}$ & 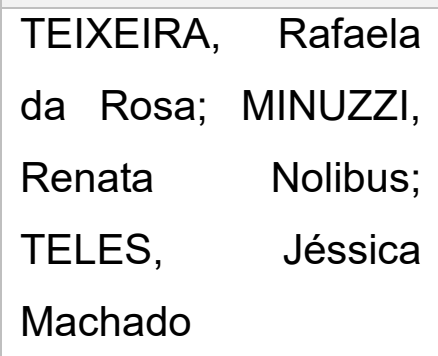 & $\begin{array}{l}\text { Cuidados de enfermagem à } \\
\text { pacientes com hipertensão } \\
\text { gestacional: um relato de } \\
\text { experiência }\end{array}$ \\
\hline 2017 & $\begin{array}{l}\text { Rev Port Med Geral } \\
\text { Fam }\end{array}$ & $\begin{array}{l}\text { FERREIRA, Sara } \\
\text { Santos, et al }\end{array}$ & $\begin{array}{l}\text { Ácido acetilsalicílico na } \\
\text { prevenção da pré-eclâmpsia: } \\
\text { uma revisão baseada na } \\
\text { evidência }\end{array}$ \\
\hline 2015 & $\begin{array}{l}\text { Ciência } \\
\text { Enfermeria }\end{array}$ & $\begin{array}{l}\text { KRAUZER, Ivete } \\
\text { Maroso, et al. }\end{array}$ & $\begin{array}{l}\text { Sistematização da } \\
\text { assistência de enfermagem } \\
\text { na atenção básica: o que } \\
\text { dizem os enfermeiros? }\end{array}$ \\
\hline 2015 & $\begin{array}{l}\text { J. res.: fundam. } \\
\text { care. }\end{array}$ & $\begin{array}{l}\text { FERNANDES, } \\
\text { Amélia Carolina } \\
\text { Lopes }\end{array}$ & $\begin{array}{l}\text { Sistematização da } \\
\text { assistência de enfermagem } \\
\text { na prevenção de infecções } \\
\text { em unidade de terapia } \\
\text { intensiva }\end{array}$ \\
\hline 2015 & $\begin{array}{l}\text { Escola Anna Nery } \\
\text { Revista de } \\
\text { Enfermagem }\end{array}$ & $\begin{array}{l}\text { SOARES, Mirelle } \\
\text { Inácio, et al }\end{array}$ & $\begin{array}{l}\text { Sistematização da } \\
\text { assistência de enfermagem: } \\
\text { facilidades e desafios do } \\
\text { enfermeiro na gerência da }\end{array}$ \\
\hline
\end{tabular}




\begin{tabular}{|l|l|l|l|}
\hline 2016 & Rev. Interd & MONTEIRO, M. M., et & $\begin{array}{l}\text { assistência } \\
\text { Emergências obstétricas: } \\
\text { al. }\end{array}$ \\
& $\begin{array}{l}\text { características de casos } \\
\text { atendidos por serviço móvel } \\
\text { de urgência }\end{array}$ \\
\hline
\end{tabular}

Fonte: Autorialità propria (2021)

\subsection{CARATTERISTICHE DELL'IPERTENSIONE IN GRAVIDANZA}

In questa ricerca avremo enfasi su una patologia che è la seconda causa principale di morte perinatale in Brasile, che è l'ipertensione in gravidanza, in cui comporta diversi rischi per la madre e il bambino, quindi è necessario seguire regolarmente questi pazienti e guidare i cambiamenti nello stile di vita e nella dieta (ARAÚJO et al., 2017).

La pressione arteriosa (PA) presenta alterazioni nell'interazione di fattori neuroumorali, comportamentali e ambientali. L'ipertensione (HA) può essere concettualizzata come una sindrome di origine multifattoriale, considerata come uno dei maggiori problemi nell'area della salute pubblica essendo valutata come un grave fattore di rischio per le malattie cardiovascolari (ARNALDO; CARDOZO, 2021).

Le donne che usano sigarette e contraccettivi, di età superiore ai 30 anni, finiscono per essere le più colpite, perché sono sottomesse ad altre malattie polmonari. Le donne ricevono protezione a causa di eventi cardiovascolari che si verificano prima della menopausa, negli uomini si presentano solo dopo i 30 anni (SOARES et al., 2015).

Studi a lungo termine hanno identificato che non sono diversi dagli uomini per quanto riguarda la risposta antipertensiva alla pressione sanguigna; tuttavia, è di fondamentale importanza evitare l'uso di inibitori enzimatici dell'angiotensina da usare $(E C A)$ e antagonisti del recettore dell'angiotensina II nelle donne in età fertile e che non utilizzano metodi anticoncetti sicuri comprovati (SANTOS; NETO, 2016). 
Le cause dell'ipertensione sono associate a una vita sedentaria, con un'assunzione inadeguata di alimenti, come l'eccesso di sodio e grassi o il consumo di alcol, e persino il fumo, che è la causa dell'insufficienza cardiaca. II fatto è che l'ipertensione è un potente contributo alle malattie cardiovascolari, attaccando e sovraccaricando il cuore fino a quando un giorno fallisce (CABRAL, 2019).

L'ipertensione in gravidanza è la seconda causa di morte materna e perinatale in Brasile secondo i dati del Ministero della Salute. Questo dimostra la necessità di conoscere e controllare questa patologia. In gravidanza l'organismo della donna subisce diverse trasformazioni per poter sviluppare il feto che sarà riparato per circa 280 giorni o circa 9 mesi. In Brasile, la popolazione dovrebbe raggiungere il segno di 228 milioni di abitanti nel 2025 (CABRAL, 2019).

II tasso di mortalità secondo IBGE nel 2016 è di 6,6 nati vivi per mille abitanti. Alla luce di questi dati, è evidente la necessità di sviluppare ricerche e progettazione per migliorare questo indice allarmante, dalla mappatura dei luoghi che hanno il più alto rischio gestazionale, che può intensificare le cure per le donne in gravidanza e quindi migliorare il tasso di mortalità (ENDRINGER; CRUZ, 2017).

\subsection{ASPETTI GENERALI DELLA PRE-ECLÂMPSIA}

Non è noto con certezza quando sono stati identificati i primi casi di questa patologia, ma ciò che è noto è che è stato descritto dai tempi in cui sono iniziate a essere fatte le registrazioni, il suo verificarsi è stato attribuito a cattivi spiriti, cattivo genio e, più recentemente, assunzione di sodio e aumento di peso durante la gravidanza (ENDRINGER; CRUZ, 2017).

Si nota che questa sindrome ha causato gravi disturbi nelle giovani donne in gravidanza correlati con la prima gravidanza ed è caratterizzata come la principale causa di mortalità materna in Brasile. Nel 1998, la tossiemia in gravidanza era responsabile del $21 \%$ dei decessi ostetrici diretti (SANTOS; BRASILIANO, 2018).

La preeclampsia è caratterizzata come una delle sindromi ipertensiva che possono colpire le donne durante la gravidanza, durante il parto e/o nel periodo postpartum fino RC: 99509

Link di accesso: https://www.nucleodoconhecimento.com.br/salute/rischi-di-ipertensione 
a 10 giorni, tanto che è ancora una patologia che, se non adeguatamente diagnosticata e trattata, può causare gravi danni e persino portare alla morte della donna incinta e/o del parto (SARMENTO et al., 2020).

È caratterizzata come una malattia sistemica che deriva dalla $20^{\text {a }}$ settimana di gestazione dalla triade: ipertensione, edema e proteinuria e può avere la sua condizione aggravata per eclampsia, in presenza di convulsioni, e/o evolvere in sindrome HELLP (SANTOS; BRASILIANO, 2018).

Le cause della preeclampsia sono ancora sconosciute, tuttavia, ciò che è noto è che alcune donne in gravidanza hanno maggiori probabilità di essere colpite da questa patologia. Nell'antichità si riferivano a cattivi geni, aumento di peso e assunzione eccessiva di sale (FERREIRA et al., 2017).

Oggi non si conoscono ancora con certezza le ragioni causali e scatenanti della preeclampsia, ciò che si sa è che ha multicausali: predisposizione familiare e genetica, endocrino-metabolica, compresa l'alterazione della produzione di prostaglandine, ischemia utero placentare, immunologica, presenza di tessuto trofoblastico, tossine nel sangue materno, causando vasocostrizione e placenta difettosa (SARMENTO et al., 2020).

La preeclampsia ha diverse eziologie che possono provenire da cause ereditarie e/o acquisite dovute allo stile di vita del paziente o alle condizioni sociali e ambientali. Tuttavia, ciò che è noto è che le condizioni sociali, insieme a una vita sedentaria, una cattiva alimentazione e la mancanza di informazioni di solito derivate da una bassa scolarità, favoriscono il rischio imminente dell'insorgenza della preeclampsia, oltre ad altri (TEIXEIRA; MINUZZI; TELES, 2016).

La sindrome ipertensiva si presenta inizialmente come preeclampsia lieve, poi progredisce in preeclampsia grave, eclampsia, sindrome di Hellp, CIVD (coagulazione intravascolare disseminata) e infine morte. E questi sono gli stadi di gravità della sindrome ipertensiva, tuttavia, non ha necessariamente bisogno di passare attraverso tutte le fasi, e può evolvere da una lieve preeclampsia alla Sindrome di Hellp (FERREIRA et al., 2017).

RC: 99509

Link di accesso: https://www.nucleodoconhecimento.com.br/salute/rischi-di-ipertensione 
Altre complicanze della preeclampsia sono: distacco prematuro della placenta a seguito di lesione placentare nel sito di impianto, edema polmonare acuto, insufficienza renale acuta dovuta a glomerulopatia installata ed emorragia cerebrale (TEIXEIRA; MINUZZI; TELES, 2016).

I radicali liberi $\mathrm{O} 2$ sono identificati con cellule adipose che sono endotelio, solubili nel sangue materno e molto vicine all'endotelio vascolare. Riducono la produzione di prostaciclina e ossido nitrico che sono vasi dilatati e aumentano la resistenza vascolare, causando un aumento della pressione sanguigna (FERREIRA et al., 2017).

L'unica alternativa per controllare la preeclampsia e prevenirla dall'eclampsia è il corretto follow-up delle cure prenatali durante la gravidanza. I pazienti con preeclampsia lieve devono optare per il riposo, misurare sempre la frequenza della pressione sanguigna e aderire a una dieta a basso contenuto di sale (KRAUZ et al., 2015).

I farmaci antipertensivi e anticonvulsivanti sono indicati per il controllo dell'eclampsia con maggiore gravità, che può richiedere l'anticipazione del parto. E la malattia tende a regredire spontaneamente con la rimozione della placenta (FERNANDES, 2015).

Pertanto, secondo Varella (2020, p. 1), è importante seguire alcune raccomandazioni:

- Se dirigir ao ginecologista antes de engravidar para uma avaliação clínica e início da administração de ácido fólico;

- Importante estar presente em todas as consultas previstas no pré-natal;

- Qualquer desatenção e a ausência de sintomas podem fazer com que uma forma leve de pré-eclâmpsia evolua com complicações;

- Realizar exercícios físicos de acordo com a fase da gestação;

- Reduzir a quantidade de sal nas refeições, não fumar e principalmente não ingerir álcool durante a gravidez (VARELLA, 2020, p. 1).

Il trattamento consiste nel riposo a letto (lato sinistro), in un luogo arioso e tranquillo, con l'uso di droghe, con l'intenzione di ridurre al minimo il peggioramento e posticipare il travaglio prematuro, quando possibile, cioè quando non vi è alcun rischio imminente 
per la madre e il feto. Tuttavia, quando ciò non è possibile, l'indicazione è l'interruzione immediata della gravidanza, indipendentemente dal periodo gestazionale (FERREIRA et al., 2017).

\subsection{ASSISTENZA INFERMIERISTICA A LIVELLO DI ASSISTENZA SANITARIA FEMMINILE}

La Sistematizzazione dell'Assistenza Infermieristica (SAE) mira a un'assistenza in modo ampio e dinamico, con una pianificazione che coinvolge i processi infermieristici nel loro complesso, la cui cura conta sulla partecipazione armonica di più professionisti insieme alla famiglia e al paziente, costruendo un ambiente favorevole al recupero con successo e con meno usura sia per il paziente-famiglia che per il team (SOARES et al., 2015).

L'assistenza infermieristica mira anche allintegrazione nella società, dove il suo obiettivo principale è quello di fornire qualità della vita alle persone, al fine di portare a una migliore e più ampia cura dei loro bisogni specifici, dando priorità alle persone che sono sotto assistenza e attenzione infermieristica, cioè coloro che già seguono (MONTEIRO et al., 2015).

"Planned Nursing" cerca di svolgere una guida professionale per quanto riguarda l'elaborazione di una pianificazione sistematica dell'assistenza infermieristica. La serie di principi qui coordinati è attribuita alla nomenclatura del "Piano Terapeutico Infermieristico", un piano che compone l'intero processo di azioni e decisioni volte a programmare l'assistenza infermieristica. (SOARES et al., 2015, p. 50).

Resta inteso che l'assistenza infermieristica è tutta la cura o la tecnica dispensata a un paziente e ogni procedura che riduce o minimizza il dolore. Quando l'infermiere si prende cura di un paziente, dispensa non solo attenzione, ma si connette al cliente per ridurre il loro dolore, fornendo così il loro recupero, riducendo le loro lesioni e incoraggiando il loro miglioramento sia fisico che mentale, attraverso l'azione insieme al team sanitario e alla famiglia (MONTEIRO, et al., 2015). 
Tuttavia, pianificare l'assistenza infermieristica è una missione che presenta un certo grado di complessità a causa di diversi fattori: uno di questi fattori è che il processo di pianificazione in infermieristica coinvolge la partecipazione armonica di più persone, come il paziente, l'infermiere e il suo team e i membri del team sanitario; un altro motivo è l'indispensabilità dell'uso del ragionamento, del processo decisionale, delle prestazioni e della documentazione; oltre a questi, un'altra ragione preponderante è legata ai costanti cambiamenti nella vita che influenzano anche i piani più migliorati (SOARES et al., 2015).

Si osserva che per eseguire una buona assistenza infermieristica è necessario prendere in considerazione diversi fattori, uno dei principali fattori è la capacità degli infermieri di condurre il lavoro insieme al team sanitario, prendendosi cura dei paradigmi del paziente e della sua famiglia. Utilizzando la sua capacità di trasmettere informazioni e, d'altra parte, di saper ascoltare, e questo costituisce una componente fondamentale per un buon professionista infermieristico (MONTEIRO et al., 2015).

L'infermiere curante fa parte di un team multidisciplinare che mira ad azioni preventive e curative all'interno della comunità attraverso la promozione di politiche sanitarie volte a ridurre le notifiche di danno, attraverso la conoscenza delle cause e dei rischi a cui la popolazione è esposta e, allo stesso tempo, sensibilizzare gli enti pubblici alle azioni che dovrebbero essere attuate (KRAUZER, 2015).

Quando si esegue l'anamnesi in un determinato cliente, è necessario adottare uno sguardo ponderato e olistico mirando non solo alla patologia sottostante o a quella che è in evidenza, ma anche a cercare di capire le ragioni per cui è emersa, al fine di cercare una soluzione per il paziente (SOARES et al., 2015).

In questa raccolta di dati, gli infermieri dovrebbero elaborare piani di assistenza sistematizzati al fine di promuovere e recuperare la salute, che era stata scossa, mirando a una diagnosi corretta. Per Carpenito apud Fernandes, et al (2015, p. 1.581):

O diagnóstico de enfermagem baseia-se tanto nos problemas reais (voltados para o presente) quanto nos problemas potenciais (voltados para o futuro), que podem ser sintomas de disfunções fisiológicas, comportamentais, psicossociais ou espirituais.

RC: 99509

Link di accesso: https://www.nucleodoconhecimento.com.br/salute/rischi-di-ipertensione 
Resta inteso che la diagnosi infermieristica si divide in due momenti: una vera e propria diagnosi che si caratterizza per le manifestazioni definite già esistenti nell'individuo, cioè la mobilità fisica compromessa, correlata alla lesione degli arti inferiori. II secondo è che la diagnosi di rischio è caratterizzata da fattori di rischio a cui l'individuo è esposto, vulnerabile sia dall'ambiente che dalla propria immunità fisica, cioè dal rischio di infezione correlato al momento del ricovero in ospedale (KRAUZER, 2015).

\subsection{ASSISTENZA INFERMIERISTICA E AZIONI DI PREVENZIONE PREECLAMPSIA}

Durante le cure prenatali vengono richiesti alcuni test e attraverso questi possono essere rilevate le patologie acute o croniche che sono dirette al trattamento in modo da non danneggiare il ciclo gestazionale e diminuire i tassi di morbilità e mortalità materna e infantile (ARNALDO; CARDOZO, 2021).

La consultazione prenatale deve avvenire all'inizio della gravidanza, poiché è possibile eseguire una valutazione più dettagliata delle condizioni materne e fetali. Anche se la gravidanza è considerata un normale processo fisiologico, si verificano diversi tipi di cambiamenti nel corpo della donna e questo causa una distanza più breve tra salute e malattia rispetto a quando non è incinta. La prevenzione o almeno la diagnosi precoce dei segni anomali, e immediatamente un trattamento efficace, possono agire come strategia per ridurre al minimo vari tipi di complicanze associate al parto, non solo durante il periodo antepartum, ma anche durante il travaglio (ENDRINGER; CRUZ, 2017).

È noto che l'operatore sanitario dovrebbe essere a conoscenza di qualsiasi segno che possa indicare una situazione che compromette l'assistenza prenatale, per questo motivo l'infermiere dovrebbe eseguire tutti i passaggi, oltre alla richiesta di test di laboratorio (SANTOS; BRASILEIRO, 2018).

L'allattamento deve sempre prestare attenzione per tutto il periodo gestazionale in modo che eventuali eventi non pianificati non si manifestino durante questo evento. Le azioni di promozione della salute e di prevenzione dei rischi durante la gravidanza

RC: 99509

Link di accesso: https://www.nucleodoconhecimento.com.br/salute/rischi-di-ipertensione 
devono concentrarsi sul servire questa clientela molto più specifica che richiede tutte le cure. In questo contesto, l'assistenza prenatale, come raccomandato dal Ministero della Salute, funge da modo principale per generare la sicurezza che i clienti e gli operatori sanitari devono avere rispetto al consolidamento di una gravidanza priva di rischi e complicanze (MONTEIRO et al., 2016).

In questo pregiudizio, la consultazione infermieristica, il follow-up della donna incinta durante le cure prenatali, l'attenzione a qualsiasi manifestazione clinica al di fuori del pianificato e la funzione di consulenza come fondamentale e necessità di ricevere un monitoraggio adeguato. D'altra parte, richiamano l'attenzione sulle complicanze legate allipertensione arteriosa, alla presenza di emorragie, al sanguinamento e ai segni di preeclampsia ed eclampsia, come fari di complicanze più profonde e mortalità (SANTOS; BRASILEIRO, 2018).

La preeclampsia ha la sua insorgenza lenta e graduale con gravi complicazioni per la madre e il feto, quindi è necessario che ci sia una cura prenatale di buona qualità che preveda azioni preventive e l'identificazione di casi predisponenti al fine di diagnosticare il più presto possibile, per iniziare il trattamento profilattico. Gli eventi dovrebbero anche essere registrati nelle cartelle cliniche in modo che queste informazioni non vengano perse e facilitino le cure future (MONTEIRO et al., 2016).

L'infermiere professionale dovrebbe eseguire già nella prima consultazione con la donna incinta, un'anamnesi completa ottenendo quante più informazioni rilevanti per la loro salute attuale e precedente, oltre alla storia familiare, e per questo dovrebbe richiedere test di routine, supporto vitaminico, guida sulla loro dieta, compilando la loro carta di gravidanza, controllare la loro scheda di vaccinazione e programma per la prossima consultazione (ENDRINGER; CRUZ, 2017).

L'assistenza infermieristica nel periodo prenatale è di fondamentale importanza perché monitora e identifica possibili cambiamenti nel suo periodo iniziale, e inoltra a una valutazione più accurata in un'unità di riferimento, con questo corretto processo decisionale può fare la differenza, e fornire alla donna incinta una maggiore sicurezza e caratterizzare una vera cura preventiva, vale la pena ricordare che il professionista

RC: 99509

Link di accesso: https://www.nucleodoconhecimento.com.br/salute/rischi-di-ipertensione 
infermieristico è un protagonista di incalcolabile importanza nell'assistenza prenatale, perché è proprio perché ha il più grande legame con le donne incinte (SANTOS; BRASILEIRO, 2018).

\section{CONSIDERAZIONI FINALI}

Lo studio, costruito da una revisione della letteratura specializzata, ha permesso un'analisi sulla prevenzione della preeclampsia nelle cure primarie e, a questo scopo, ha fatto una breve storia sull'incidenza di questa patologia, nonché sui suoi concetti e caratteristiche.

Inoltre, è stato opportuno descrivere i contributi dell'assistenza infermieristica nell'assistenza primaria. Pertanto, resta inteso che per eseguire una buona assistenza infermieristica è necessario tenere conto di diversi fattori, uno dei fattori principali è la capacità degli infermieri di condurre il lavoro insieme al team sanitario, prendendosi cura dei paradigmi del paziente e della sua famiglia. Utilizzare la sua capacità di trasmettere informazioni e d'altra parte saper ascoltare, e questo costituisce componenti di base per un buon professionista infermieristico

La preeclampsia, sebbene sia una patologia o una sindrome che può colpire le donne in uno stato di gravidanza, è ancora caratterizzata come un problema di salute pubblica, nonostante l'intero sviluppo della scienza, anche nell'area infermieristica e nell'area medica, proprio il motivo scatenante dello studio, quale parte del problema proposto nell'introduzione, che, secondo i ricercatori, è stato trattato in modo approfondito, in modo che i dati ottenuti fossero coerenti e i risultati da essi possano essere generalizzati all'intera popolazione studiata.

Pertanto, utilizzando articoli che sono stati trovati incentrati sul tema di questo studio, suscita la necessità di fare molte altre pubblicazioni sulla prevenzione della preeclampsia. La maggior parte degli studi si concentra più sul ruolo degli infermieri nell'umanizzazione del contatto professionale / utente al momento del dolore acuto o cronico nei servizi di emergenza che sulla prevenzione della preeclampsia nelle cure primarie.

RC: 99509

Link di accesso: https://www.nucleodoconhecimento.com.br/salute/rischi-di-ipertensione 
Quindi, quali sono le azioni infermieristiche in Primary Care in considerazione dei rischi di ipertensione durante la gravidanza? In risposta al problema, vale la pena notare che il professionista infermieristico che lavora in un'unità sanitaria dovrebbe tenere lezioni al fine di aumentare la consapevolezza sull'importanza dell'assistenza prenatale il più rapidamente possibile per garantire una gravidanza sana e una buona evoluzione del concetto e del travaglio.

In questo senso, si conclude che le principali condotte utilizzate non solo dagli infermieri, ma dall'intero team che lavora nelle cure primarie, dovrebbero essere incentrate sul processo di accoglienza, incentrato su azioni che possono essere determinanti nella prevenzione della preeclampsia. Pertanto, è estremamente importante pubblicare più studi incentrati sulla prevenzione della preeclampsia nelle cure primarie.

\section{RIFERIMENTI}

ARAÚJO, Isabella Félix Meira, et al. Síndromes hipertensivas e fatores de risco associados à gestação. Rev enferm UFPE on line., Recife, 11(Supl. 10):4254-62, out., 2017.

ARNALDO, Mariany de Freitas. CARDOZO, Maryanne Neuraide Freire. Assistência de enfermagem às gestantes hipertensas na prevenção da prematuridade: revisão bibliográfica. Revista científica multidisciplinar núcleo do conhecimento. Ano 06, Ed. 02, Vol. 02, pp. $108-125$. Fevereiro de 2021.

CABRAL, Gustavo Pereira. Cuidados de enfermagem a mulheres com doença hipertensiva específica da gestação: uma revisão integrativa. Trabalho de Conclusão de Curso. Uruguaiana, 2019.

ENDRINGER, Deyvid Dantas; CRUZ, Monielle Lima. Representatividade do enfermeiro na assistência a gestantes com pré-eclâmpsia. International Nursing Congress. May 9-12, 2017. 
FERNANDES, Amélia Carolina Lopes. Sistematização da assistência de enfermagem na prevenção de infecções em unidade de terapia intensiva. J. res.: fundam. care. online 2015. out./dez. 6(4):1580-1589.

FERREIRA, Sara Santos, et al. Ácido acetilsalicílico na prevenção da pré-eclâmpsia: uma revisão baseada na evidência. Rev Port Med Geral Fam 2017;33:118-32.

FONSECA, J. J. S. Metodologia da pesquisa científica. Fortaleza: UEC, 2002.

GIL, Antônio Carlos. Como elaborar projetos de pesquisa. 4. ed. São Paulo: Atlas, 2007.

KRAUZER, Ivete Maroso, et al. Sistematização da assistência de enfermagem na atenção básica: o que dizem os enfermeiros? Ciência Y Enfermeria XXI (2), 2015.

LAKATOS, Eva Maria; MARCONI, Marina de Andrade. Fundamentos da metodologia científica. 7. ed. São Paulo: Atlas, 2010.

MELO, Wyara Ferreira, et al. A hipertensão gestacional e o risco de pré-eclampsia: revisão bibliográfica. REBES - Revista Brasileira de Educação e Saúde (Pombal PB, Brasil), v. 5, n. 3, p. 07-11, jul-set, 2015.

MONTEIRO, M. M., et al. Emergências obstétricas: características de casos atendidos por serviço móvel de urgência. Rev. Interd. v. 9, n. 2, p. 136-144, abr/mai/jun. 2016 143.

OLIVEIRA, Leilyanne de Araújo Mendes, et al. Cuidados de enfermagem a gestante com síndrome hipertensiva: revisão integrativa. Brazilian Journal of Surgery and Clinical Research - BJSCR. Vol.23,n.2,pp.159-164 (Jun - Ago 2018).

SANTOS, Keilane Carvalho. BRASILEIRO, Marislei Espíndula. Enfermagem e os cuidados Emergenciais na doença hipertensiva específica na gravidez. Revista Científica Multidisciplinar Núcleo do Conhecimento. Ano 03, Ed. 11, Vol. 08, pp. 17-26 Novembro de 2018. 
SANTOS, Joelma Oliveira Silva; NETO, Thiago Paulo de Almeida. Atuação do enfermeiro na redução da ocorrência da pré-eclâmpsia: uma revisão integrativa. Revista Científica da FASETE 2016.1.

SARMENTO, Rayani Silva, et al. Pré-eclâmpsia na gestação: ênfase na assistência de enfermagem. Enfermagem Brasil 2020;19(3):261-267.

SILVA, E.L. da; MENEZES, E.M. Metodologia da pesquisa e elaboração de dissertação. Florianópolis: Laboratório de Ensino à Distância da UFSC, 2001.

SOARES, Mirelle Inácio, et al. Sistematização da assistência de enfermagem: facilidades e desafios do enfermeiro na gerência da assistência. Escola Anna Nery Revista de Enfermagem 19(1) Jan-Mar 2015.

TEIXEIRA, Rafaela da Rosa; MINUZZI, Renata Nolibus; TELES, Jéssica Machado. Cuidados de enfermagem à pacientes com hipertensão gestacional: um relato de experiência. XII Semana de Extensão, Pesquisa e Pós-Graduação SEPesq - 24 a 28 de outubro de 2016

Inserito: Agosto 2021.

Approvato: Settembre 2021. 